\title{
IOT AND AI IN HEALTHCARE: A SYSTEMATIC LITERATURE REVIEW
}

\author{
Rushabh Shah, BentleyUniversity, sha1_rush@bentley.edu \\ Alina Chircu, Bentley University, achircu@bentley.edu
}

\begin{abstract}
Recent developments in technology and connectivity have led to the emergence of Internet of Things (IoT) and Artificial Intelligence (AI) applications in many industries. This paper studies the impacts of technologies such as IoT and AI in healthcare through a systematic review of 75 peer-reviewed scholarly journal articles. The analysis reveals exponential growth in the number of articles published in the last decade, a wide variety of publication outlets, a large number of authors, and many conceptual and design science papers, all indicating an emerging field with great publication potential in future years. The analysis further reveals that applications of these technologies are being studied worldwide, not just in the USA but also in many countries in Europe and Asia. The paper also highlights key insights for the top application categories, which include wearables and connectivity, disease detection and treatment, patient care, and sensor networks, and identifies gaps and future research directions related to technology design and acceptance, regulations for data security and privacy, and systems efficacy and safety.
\end{abstract}

Keywords: Artificial Intelligence (AI), Healthcare, Internet of Things (IoT), Systematic literature review

\section{INTRODUCTION}

Recent developments in technology and connectivity have led to the emergence of Internet of Things (IoT) and Artificial Intelligence (AI) applications in many industries. IoT is a set of technologies that enable objects everyday consumer products or industrial machines - to connect with one another and with the internet, communicate data about their attributes (such as working condition, temperature, movement, position, etc.), and provide "instant data analysis and, ideally, 'smart' action" (Daecher et al., 2018). IoT is projected to create value in all industries though a variety of industrial, consumer, and public sector applications (Daecher et al., 2018). The rise of IoT has resulted in the re-emergence of AI, a collection of technologies and systems able to "sense their environment, think, learn, and take action in response to what they're sensing and their objectives" (Verweij et al. 2017). IoT plays a significant role in collecting and monitoring data, whereas AI is responsible for analyzing the growing amounts of data and taking action based on what it learns from the data. According to recent predictions, IoT and AI could have huge economic impact, adding trillions of dollars to the global economy - $\$ 14.2$ trillion for industrial IoT applications and \$15.7 trillion for AI applications by 2030 (Purdy and Davarzan, 2015; Verweij et al. 2017).

One industry where IoT and AI, individually or together, are making significant impacts is the healthcare industry, which is constantly under pressure to reduce costs while addressing a rapidly growing unhealthy population. These technologies can help healthcare organizations tap into the potential of an increasingly interconnected and responsive world. If the industry is able to generate "greater interconnectivity in a single ecosystem, there will be significant benefits to patients, physicians, payers and drug developers" (King 2017). IoT devices such as smart pills, wearable monitors, and sensors allow healthcare practitioners to continuously collect data, and AI systems can help analyze this data to detect changes in a patient's condition, suggest treatment options, and identify trends, thus supporting patient adherence, improving patient outcomes, and accelerating discovery of and access to new treatments (Daecher et al., 2018; King 2017; Verweij et al. 2017).

\section{RESEARCH METHODOLOGY}

This paper studies the impacts of two technologies - IoT and AI - on healthcare through a systematic review of the literature. Systematic literature reviews, also called PRISMA style reviews, involve systematically searching for 
articles on a given topic in various library databases or through other sources, screening the articles for appropriateness, further evaluating the full text articles for eligibility, and conducting quantitative and qualitative analyses (Moher et al., 2009; Pickering and Byrne, 2014). This methodology "uses systematic and explicit methods to identify, select, and critically appraise relevant research, and to collect and analyze data from the studies that are included in the review" (Moher et al., 2009), and is designed to be comprehensive and reproducible, in contrast to the more subjective narrative review process (Pickering and Byrne, 2014). While this methodology was originally developed for healthcare reviews and meta-analyses (Moher et al., 2009), its systematic aspects are proving useful for a variety of natural and social sciences fields (Pickering and Byrne, 2014), including information systems (Wimmer et al., 2017)

To identify articles for this review, three large library databases (Academic Search Premier, Business Source Premier, and Medline) were searched in February 2018 for articles containing relevant keywords (("internet of things" or "IoT" or "artificial intelligence" or "cognitive computing") and ("healthcare" or "medical")) in the article abstract, resulting in 1448 articles. Filtering the articles to full-text resulted in 552 articles, and further restricting the results to scholarly, peer-reviewed journal articles yielded 205 articles published from 1984 to 2018. These results were then limited to articles published from 2007 to 2018, capturing the research published in the past decade 20072017 as well as the most recent articles published in January or February 2018 and indexed in the databases at the time of the search. This resulted in 152 articles, which were further evaluated for eligibility based on their abstract (or, if needed, based on the full text). 77 articles that did not directly address the research topics were eliminated, resulting in a final list of 75 articles retained for analysis.

\section{ANALYSIS AND RESULTS}

The quantitative analysis of the article meta-data identified interesting insights regarding publication trends. As Figure 1 shows, there is an upward trend in the number of articles being published each year from 2007 - 2017, with the number of articles growing exponentially since 2013 and reaching 14 articles in 2016 and 30 articles in 2017. This growth could be attributed to the increasing availability and adoption of IoT and AI technologies over the last few years, which likely resulted in increasing interest from researchers. In addition, given the current hype around $\mathrm{AI}$ and IoT, and the four articles published in the first two months of 2018, the number of articles can be expected to reach similar or even higher levels in future years.

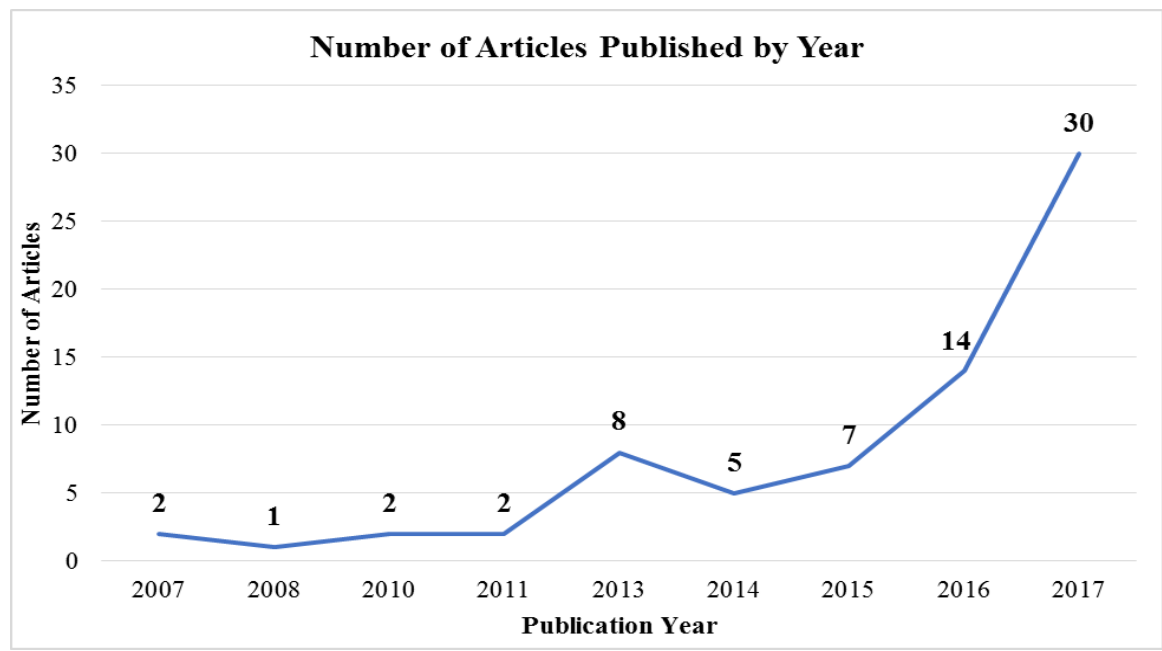

Figure 1: Articles by Publication Year

Note: Four 2018 articles not included.

The meta-data analysis also suggests that research on technologies such as AI and IoT in healthcare is published in a wide variety of journals, as shown in Table 1. The top journal by number of articles (9) is Sensors, which publishes articles focused on technical solutions and their uses and impacts. While some of these articles may seem highly 
technical, they provide unique insight into the development of IoT or AI-enhanced products, and the importance of each of these devices in creating a connected ecosystem. The list of other journals with multiple articles suggests other popular areas of focus for applications of IoT or AI in healthcare, including computer systems, biomedical research, sensor networks, science and medicine in general, as well as specialized medical areas (laboratory medicine, pathology, orthopedics) and technical areas (engineering, neural networks). Furthermore, 44 journals covering a wide variety of topics published one article each. Overall, there seemed to be a balanced blend of journals focusing on the technical aspects and application/benefits of these technologies.

In addition, the analysis of subject classification for each article reveals that, in addition to the subjects that mirror the search keywords (Artificial Intelligence, Internet of Things, Medical Technology, and Medical Care), top subjects included Medical, Dental, and Hospital Equipment (16\% of the 75 articles), Surgical and Medical Instrument Manufacturing (13\%), Other Electronic and Precision Equipment Repair and Maintenance (12\%), and Surgical Appliance and Supplies Manufacturing (11\%). This keyword analysis suggests most of the articles are focused on the design and use of medical equipment that incorporates technologies such as IoT or AI. Furthermore, the analysis of article authors shows most authors have only published one article, with just one author publishing two articles. This shows that this field of research is relatively young, attracting numerous researchers, none of whom have a disproportionate impact on the field in terms of journal output.

Table 1. Number of Articles Published, by Journal

\begin{tabular}{|l|l|}
\hline \multicolumn{1}{|c|}{ Journal Name } & Number of Articles \\
\hline Sensors & 9 \\
\hline Computers in Industry & 4 \\
\hline Biomedical Research & 4 \\
\hline Expert Systems with Applications & 3 \\
\hline International Journal of Distributed Sensor Networks & 3 \\
\hline MLO: Medical Laboratory Observer & 2 \\
\hline PLoS ONE & 2 \\
\hline Neural Computing \& Applications & 2 \\
\hline EE: Evaluation Engineering & 2 \\
\hline Archives of Pathology \& Laboratory Medicine & 2 \\
\hline Acta Orthopaedica & 2 \\
\hline Other journals (each with one article) & 40 \\
\hline
\end{tabular}

To further analyze the 75 articles selected for this review, the articles were coded based on the type of methodology used, geographical location of the research, and type of medical problem addressed in the paper. The analysis of these codes reveals that most articles (33) are conceptual papers addressing the theoretical background of healthcare applications and proposing various conceptual frameworks. The second biggest category of articles (25) focuses on the design of systems (such as expert systems for disease diagnosis or sensor-based monitoring systems for medical treatment or assisted living). The remaining categories are much smaller by comparison: case studies (7), reviews of technologies, systems, applications, or literature (6), and quantitative analyses (4). In terms of geography, the articles focus mostly on applications in Europe, USA and Asia (with 26, 23 and 22 articles, respectively), and less on other parts of the world (4 articles). In addition, the top medical problems discussed in the papers reviewed include health monitoring (16 articles), disease detection/treatment (15 articles), system accuracy/security (13 articles), and data collection/management (11 articles). Other problems covered in fewer papers include smart hospitals and medical specialization (6 articles each) and research/innovation and emergency response (4 articles each).

The articles were also coded by the type of application discussed by each article. As Figure 2 shows, the top four categories were Wearables \& Connectivity, Detection \& Treatment, Sensor Networks, and Patient Care, with 13, 12, 12 and 9 articles each, respectively. Smaller categories included Patient Care, Predictive Models/Decision Support Systems, System Management, Neural Networks, and Automation/Robotics. Note that articles that addressed unique issues not related to any of the major categories as well as articles that were too high-level were categorized as Other. Key insights from the top four categories, collectively covering $61 \%$ of the total articles, are summarized in the next subsections. 


\section{Issues in Information Systems}

Volume 19, Issue 3, pp. 33-41, 2018

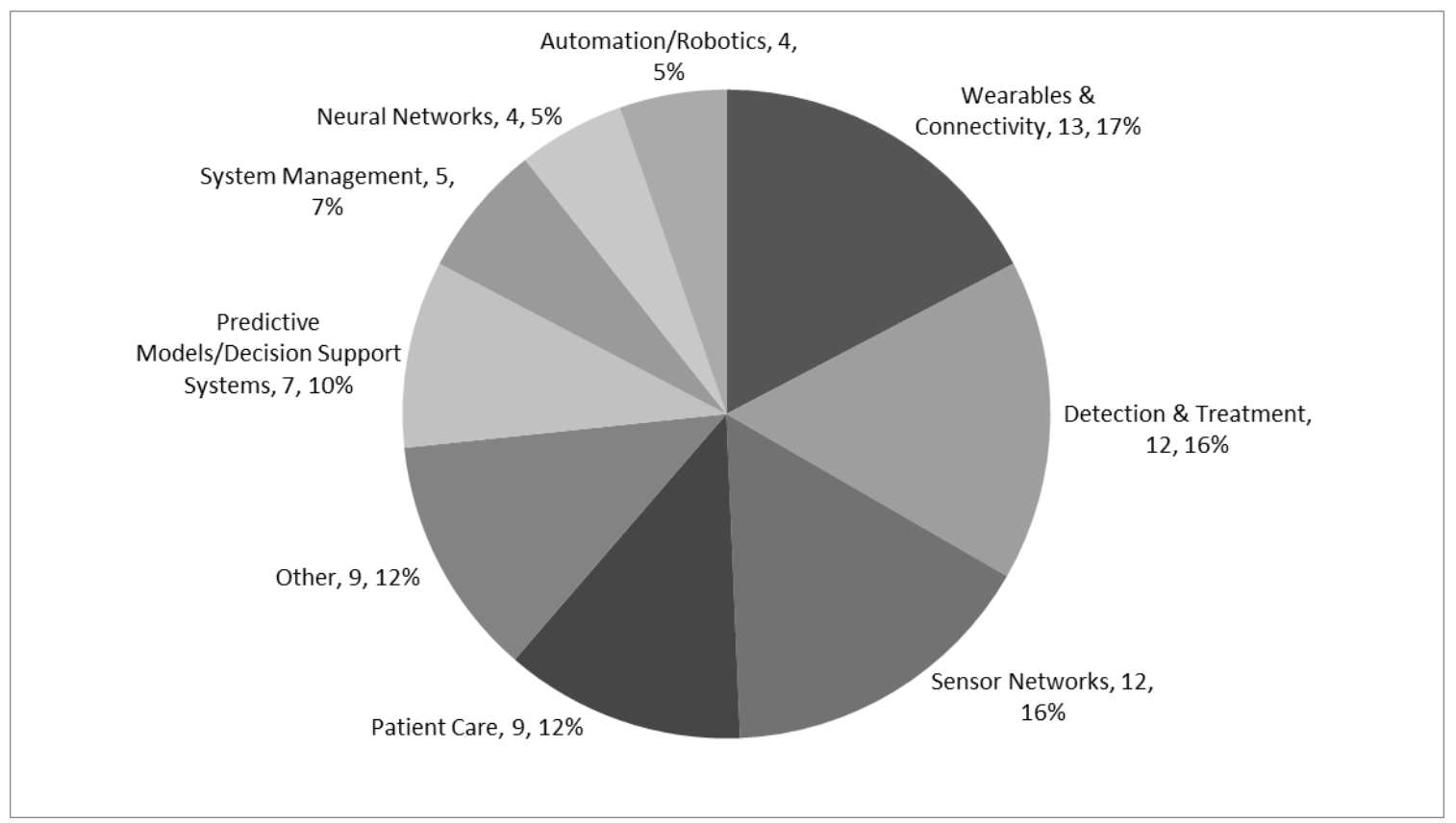

Figure 2: Articles by Type of Application

\section{Wearables \& Connectivity}

Wearable devices and connectivity between these devices and computers are key concepts driving the technological changes in healthcare. Vongsingthong and Smanchat (2014) list the key capabilities that solutions for mobile healthcare should have to be successful. These capabilities include: tracking and monitoring of patients through wearable devices, remote service through telemedicine and remote diagnosis, which are key in providing emergency detection, information management related to medication, therapy, and medical advice, and cross organizational integration of hospital information systems. One of these characteristics is analyzed in detail in Felkey and Fox (2016), who emphasize the need for medication administration to become more direct and active through mobile connectivity. They further discuss the impact of the Insightfil app that prompts and records medication regimen adherence behaviors performed by both patients and their caregivers and facilitates communication between physicians, patients, and electronic medical records.

The Wearables and Connectivity article category has a high emphasis on the use of wearable sensors in the care of the elderly. Konstantinidis et al. (2015) provide a brief overview of easily available, relatively low-cost, and easy to use controllers for elderly care interventions, including the Wii remote controller, pressure sensors, and Kinect sensors used for gaming and fitness tracking. These devices can be integrated with Smart TVs, which can become hubs for unobtrusively monitoring the status of elderly people, communicating alerts to health professionals, and helping people exercise through games, thus enabling daily life management and independent living. Furthermore, continuous monitoring, remote diagnosis and data sharing through mobile health devices and applications are critical for diseases such as strokes, sleep disorders, and epilepsy (Sun et al., 2016).

While IoT wearables can provide multitudinous benefits, the articles in this category also highlight technologyrelated risks and threats. Shahmiri (2016) discusses inherent risks associated with wearables, including hacking of devices and data, lack of clear and concise user agreement and privacy policies, lack of user data protection legislation, which could deter widespread adoption of these devices. Similarly, consumers' concerns regarding wearables health effects, accuracy and reliability, data collection and storage, and privacy are highlighted in a study by Marakhimov and Joo (2017). The authors find that consumers react strongly to these issues and that future technology needs to address them in order to be successful. It is not surprising these concerns exist, since there is a lack of regulations monitoring IoT devices. In fact, some researchers claim that although IoT has shown great promise for healthcare, it has not been as successful as expected because of the lack of strong and consistent 
regulation regarding data protection, efficacy, and safety of IoT devices (Terry, 2017). Therefore, policymakers will need to step up and provide added assurance to users in order for IoT to become a significant part of the healthcare industry.

\section{Detection \& Treatment}

Today, physicians can diagnose and treat much more diseases than in the past. However, even after years of practice, they can still struggle to make the correct diagnosis efficiently. This is where technologies such as IoT and AI can play a key role in providing reliable support for determining a diagnosis and the best course of treatment. AI technologies such as neural networks can quickly analyze the extensive amount of information available to physicians, streamline the diagnostic process, and help avoid mistakes by integrating both historical data and specific patient information (Amato et al., 2013). This is extremely important as huge amounts of healthcare data are starting to be available from a variety of sources - including IoT medical devices that can generate real-time data making human pattern detection and interpretation increasingly difficult. We know, for example, that screening processes such as X-Rays and CT scans depend on the ability of the radiologist to correctly interpret the results. However, on average, $10 \%$ of mammography screenings have inconclusive results for breast cancer, and require further biopsies which can often cause major mental and physical discomfort for patients (Keleş et al., 2011). A similar issue is faced with screening of the thyroid, where $20 \%$ of biopsies result in further surgery in order to obtain a clearer diagnosis (Paydar et al., 2016). Although not a replacement for trained physicians, AI systems can provide additional help to doctors by providing clearer images, highlighting suspicious regions, providing better analysis tools and individualized risk assessmenst for each patient, which in turn reduce the need for invasive procedures (Keleş et al., 2011, Paydar et al., 2016).

In some cases, combinations of AI and medical devices can replace expensive specialist consultations. For example, Javed et al. (2007) discuss the difficulties of diagnosing cardiovascular diseases especially in places where there are no expert cardiologists, such as rural hospitals. They develop and test a cost-effective system that combines a digital medical device with a knowledge-based system and can give a general practitioner an expert opinion regarding the potential for heart disease in his patients.

Furthermore, disease detection and monitoring can be enhanced by IoT-based systems. For example, Sood and Mahajan (2017) propose a system that uses cloud computing, wearable sensors and cloud-based healthcare services to remotely detect the rapidly-spreading Chikungunya virus, which is extremely challenging to diagnose quickly and early. Vilallonga et al. (2013) describe a personalized home-based electronic healthcare experiment comparing the use of in-home Wi-Fi enabled weighing scales versus weekly meetings for bariatric surgery patients. Interestingly, while all patients with Wi-Fi scales were seemingly happy with the use of technology and achieved weight loss, most patients indicated they would prefer face-to-face meetings with their surgeon.

Overall, most articles in the Diagnosis \& Treatment category indicate that technologies such as IoT and AI provide patients and doctors with clear benefits. However, the success of these technologies in healthcare will depend on doctors' and patients' willingness to accept the new technology. Thus, designers and implementers of these technologies should always keep usability and adoption in mind.

\section{Sensor Network}

Sensor networks provide the foundation for the design, development, and implementation of a variety of healthcare applications, many of which are discussed in other sections of this paper. Monares et al. (2014) propose a modelling approach that uses wireless sensor networks to evaluate IoT models during the design phase. The sensors enable better design and testing phases, which reduce the need to make major changes once the device is launched and results in significant cost savings. New designs for sensor networks also provide solutions to security and privacy issues. For example, Hou and Yeh (2015) introduce two secure communication protocols that facilitate single sign on for a variety of systems, servers, and devices.

Sensor networks are also the foundation for operational excellence in hospitals, creating business value in addition to the value for patients, clinicians, and caregivers discuss previously. Perry (2016) discusses how IoT sensors can be used in healthcare business operations, in areas such as material management, pharmacy rentals, process management, expense management, and human resource management. Adding sensors to everything from patient beds to nurse badges and hand sanitizers can help a healthcare facility collect and analyze data to improve supply 
chains, change processes, and update procedures. For example, the use of such a sensor network allowed a hospital to identify issues in their daily processes and tackle them before they became a bigger problem (Perry, 2016). Sensor networks can promote automation and reduce human intervention in many processes, thus reducing costs and increasing efficiency (Rizwan, 2017).

In addition, sensor networks can support the development of platforms for patients and caregivers. The Common Recognition Identification Platform (CRIP) is one such sensor-based platform proposed by Miranda et al. (2016). CRIP allows for continuous health management and well-being through the integration and interaction of sensors and people. The system was tested in a nursing home environment and results showed increased patient adherence and awareness when undergoing treatment. Another such platform is the Aging in Place (AIP) system proposed in Fattah et al. (2017), which focuses on caregivers (rather than patients), who are suffering from more stress and depression due to their caregiver status. AIP tests showed it can help increase patient autonomy by providing medication reminders through lights and speakers at home and wrist bands and mobiles outside the home. This, in turn, reduced the pressure on people living with these patients.

\section{Patient Care}

Patient care is the most important aspect in any hospital, and the implementation of patient care technology in hospitals can be key in improving the quality of care. Future smart hospitals will rely on new integrated technology, an ecosystem of platforms, and staff that is able to use the new technology to re-invent the care process and better serve patient needs (Mokhtar, 2017). After discharge, the smart hospital ecosystem can be extended through digital homes as well. This ecosystem will facilitate the constant monitoring, storing and analyzing of patient data. Robotics and AI are also emerging as key technologies in patient care and disease management, especially in the European Union, where new compliance and safety rules are in effect (Tsang et al., 2017).

While these patient care systems provide clear benefits for patients, doctors, and caregivers, there are potential ethical issue with continuous data monitoring and storage. Ludwin and Murry (2017) posit that the exponential growth of big data in healthcare raises such ethical issues. This is even more important, given the fact that patients are often monitored by numerous devices at the same time and they do not know what each device is monitoring and what data is being saved without their knowledge. The article finds that there is significant discussion in the literature to identify ways of going beyond current laws and allowing for certain exemptions with regards to consent as long as regular monitoring of data is implemented, but that other authors argue for more patient autonomy instead.

\section{DISCUSSION AND CONCLUSIONS}

This paper studies the impact of technologies such as IoT and AI in healthcare, using a systematic literature review of 75 peer-reviewed scholarly journal articles identified and analyzed through a rigorous process. The analysis reveals exponential growth in the number of articles published in the last decade, a wide variety of publication outlets, and a large number of authors, all indicating an emerging field with great publication potential in future years. This conclusion is reinforced by an analysis of meta-data subjects and by the authors' own coding of article topics, which indicate that the majority of papers are either conceptual papers or papers describing the design and testing of technology-enabled applications and devices, which is expected for nascent areas of research. The analysis also reveals that applications using emerging technologies are being developed worldwide, not just in the USA but also in many countries in Europe and Asia. In addition, the results point out that technologies such as IoT and AI can improve healthcare in a variety ways, but that further developments are needed for system accuracy, security, data collection and management, and privacy protection. Last, but not least, the paper highlights key insights for the top application categories, which include wearables and connectivity, disease detection and treatment, patient care, and sensor networks. These applications have numerous potential benefits for patients, their caregivers, doctors, and hospitals. However, technology acceptance by doctors and patients and development of stronger and more consistent regulations for data security and privacy, and systems efficacy and safety are key determinants of success for healthcare applications using technologies such as IoT and AI.

This paper has several limitations. While three databases were used to search for relevant articles, it is possible that additional articles could be identified by using more databases. Searching different databases, such as IEEE Xplore, 
could identify articles that present a more technical perspective on healthcare applications, and is recommended for follow-up studies focusing on in-depth technical issues. Another limitation is focusing on full text, peer-reviewed journal articles. While this choice was made in order to understand where the most advanced research is published, future research can also include conference proceedings and working papers for a more complete view of emerging ideas in the field. For example, respected technical professional associations such as IEEE and ACM organize relevant conferences and workshops - including healthcare-focused events (such as IEEE International Conference on Healthcare Informatics or the IEEE/ACM Conference on Connected Health) as well as more generic IoT or AIfocused events where healthcare technology research can be presented (such as the IEEE Hawaii International Conference on System Sciences (HICSS)). In addition, information systems conferences such as those organized by the International Association for Computer Information Systems (IACIS) or the Association for Information Systems (AIS) could also be searched for relevant articles focusing more on the implementation and adoption aspects of emerging technologies.

This systematic literature review highlights key concepts in research on technologies such as IoT and AI in healthcare, such as benefits, adoption barriers, and privacy and safety issues, but also identifies some gaps in the literature. Among the articles reviewed in this paper, there is an emphasis on conceptual and design papers, which contain no or limited testing. Future research should focus on large-scale testing of the proposed systems and frameworks in real settings. Future studies should also focus on improving the security and privacy of IoT devices that monitor and store patient data, since the fear of leaked data has been found to be the biggest hurdle for consumer acceptance. Lastly, future studies should investigate technology ecosystems (such as those required for smart hospitals) in different technical, economic, social, and cultural environments, and provide insights on interoperability and adoption challenges in these different settings. This will help provide more nuanced insights on how technologies such as IoT and AI can improve healthcare around the world.

\section{REFERENCES}

Amato, F., López, A., Peña-Méndez, E. M., Vaňhara, P., Hampl, A., \& Havel, J. (2013). Artificial neural networks in medical diagnosis. Journal of Applied Biomedicine (De Gruyter Open), 11(2), 45-58.

Daecher, A., Cotteleer, M., \& Holdowsky, J. (2018). The Internet of Things: A technical primer. Deloitte. Available: https://www2.deloitte.com/insights/us/en/focus/internet-of-things/technical-primer.html.

Fattah, S. M. M., Nak-Myoung Sung, Il-Yeup Ahn, Ryu, M., \& Yun, J. (2017). Building IoT services for aging in place using standard-based IoT platforms and heterogeneous IoT products. Sensors (14248220), 17(10), 1 29.

Felkey, B. G., \& Fox, B. I. (2016). Is this the first adherence-focused multidisciplinary care team app? Hospital Pharmacy, 51(1), 94-95.

Hou, J., \& Yeh, K. (2015). Novel authentication schemes for IoT based healthcare systems. International Journal of Distributed Sensor Networks, 11(11), 1-9.

Javed, F., Venkatachalam, P. A., \& Hani, A. M. (2007). Knowledge based system with embedded intelligent heart sound analyser for diagnosing cardiovascular disorders. Journal Of Medical Engineering \& Technology, 31(5), 341-350.

Keleş, A., Keleş, A., \& Yavuz, U. (2011). Expert system based on neuro-fuzzy rules for diagnosis breast cancer. Expert Systems with Applications, 38(5), 5719-5726.

King, W. The 'healthcare internet of things'. Pharmaceutical Executive, 37(1), 34-35.

Konstantinidis, E. I., Antoniou, P. E., Bamparopoulos, G., \& Bamidis, P. D. (2015). A lightweight framework for transparent cross platform communication of controller data in ambient assisted living environments. Information Sciences, 300, 124-139. 
Ludwin, S. K., \& Murray, T. J. (2017). Dilemmas in medical ethics in the age of big data. Multiple Sclerosis Journal, 23(10), 1306-1308.

Marakhimov, A., \& Joo, J. (2017). Consumer adaptation and infusion of wearable devices for healthcare. Computers in Human Behavior, 76, 135-148.

Miranda, J., Cabral, J., Wagner, S. R., Pedersen, C. F., Ravelo, B., Memon, M., et al. An open platform for seamless sensor support in healthcare for the internet of things. Sensors (14248220), 16(12), 1-22.

Moher, D., Liberati, A., Tetzlaff, J., Altman, D. G., \& Prisma Group. (2009). Preferred reporting items for systematic reviews and meta-analyses: the PRISMA statement. PLoS Medicine, 6(7).

Mokhtar, A. M. (2017). The future hospital: A business architecture view. Malaysian Journal of Medical Sciences, 24(5), 1-6.

Monares, Á., Ochoa, S. F., Santos, R., Orozco, J., \& Meseguer, R. (2014). Modeling IoT-based solutions using human-centric wireless sensor networks. Sensors (14248220), 14(9), 15687-15713.

Paydar, S., Pourahmad, S., Azad, M., Bolandparvaz, S., Taheri, R., Ghahramani, Z., et al. (2016). The evolution of a malignancy risk prediction model for thyroid nodules using the artificial neural network. Middle East Journal of Cancer, 7(1), 47-52.

Perry, P. M. (2016). Understanding the internet of things. Hfm (Healthcare Financial Management), 70(10), 82-83.

Pickering, C.M., \& Byrne, J. (2014). The benefits of publishing systematic quantitative literature reviews for $\mathrm{PhD}$ candidates and other early career researchers. Higher Education Research and Development, 33, 534-548.

Purdy, M., \& Davarzan, L. (2015). The Growth Game-Changer: How the Industrial Internet of Things Can Drive Progress and Prosperity. Accenture.

Rizwan, P., M., R. B., \& K., S. (2017). Design and development of low investment smart hospital using internet of things through innovative approaches. Biomedical Research (0970-938X), 28(11), 4979-4985.

Shahmiri, S. (2016). Wearing your data on your sleeve: Wearables, the FTC, and the privacy implications of this new technology. Texas Review of Entertainment \& Sports Law, 18(1), 25-48.

Sood, S. K., \& Mahajan, I. (2017). Wearable IoT sensor based healthcare system for identifying and controlling chikungunya virus. Computers in Industry, 91, 33-44.

Sun, J., Guo, Y., Wang, X., \& Zeng, Q. (2016). mHealth for aging China: Opportunities and challenges. Aging \& Disease, 7(1), 1-15.

Terry, N. P. (2017). Will the internet of things transform healthcare? Vanderbilt Journal of Entertainment \& Technology Law, 19(2), 327-352.

Tsang, L., Kracov, D. A., Mulryne, J., Strom, L., Perkins, N., Dickinson, R., et al. (2017). The impact of artificial intelligence on medical innovation in the european union and united states. Intellectual Property \& Technology Law Journal, 29(8), 3-11.

Verweij, G., \& Rao, A. (2017). Sizing the prize. PwC.

Vilallonga, R., Lecube, A., Fort, J. M., Boleko, M. A., Hidalgo, M., \& Armengol, M. (2013). Internet of things and bariatric surgery follow-up: Comparative study of standard and IoT follow-up. Minimally Invasive Therapy \& Allied Technologies, 22(5), 304-311. 


\section{Issues in Information Systems}

Volume 19, Issue 3, pp. 33-41, 2018

Vongsingthong, S., \& Smanchat, S. (2014). Internet of things: A review of applications and technologies. Suranaree Journal of Science \& Technology, 21(4), 359-374.

Wimmer, H., Rebman Jr, C. M., \& Booker, Q. E. (2017). Evaluation of predictive analytic techniques in healthcare research: a PRISMA style review. Issues in Information Systems, 18(3), 88-99. 